\title{
Point of care ultrasound: emerging trend
}

\author{
Delores Jones* \\ Registered Diagnostic Medical Sonographer from American Registry of Diagnostic Medical Sonography, University of Phoenix, USA
}

\begin{abstract}
Healthcare is expanding and there are many industries within this field. Healthcare is a necessity and there are often not enough medical professionals to keep up with that demand. As a result of this need, many radiology modalities are trying to come up with new protocols to serve patients faster. Ultrasound has made a large impact on helping with this dilemma. Ultrasound has applications that can assist with quickly assessing a patient. One application is Point of Care Ultrasound (otherwise referred to as POCUS). Point of Care Ultrasound helps decrease patient wait times so that radiologists can look at diagnostic images quickly obtained by a medical professional to help find out what is causing certain medical symptoms of patients ("Point of care," n.d.). POCUS is being used more and more throughout the United States and is a current trend within the ultrasound industry. This article will explain what POCUS is, benefits of POCUS, and applications of POCUS.
\end{abstract}

\section{Introduction}

One of the current trends within the ultrasound industry is Point of Care Ultrasound. Point of Care Ultrasound or POCUS allows medical professionals to quickly assess specific parts of a patient. POCUS was created to help with assisting medical professionals to swiftly look at specific body parts that may be the cause of specific symptoms that are occurring within a patient. The convenience of POCUS being utilized next to patient bedside has resulted in many health facilities utilizing this procedure. Figure 1 lists a summary of the benefits of POCUS focused patient care. Note that POCUS allows reduced costs, increased patient care outcomes, and it can be performed next to a patient ("The benefits of point," 2011). The subsequent paragraphs will define what Point of Care Ultrasound is while discussing various applications of POCUS (Table 1).

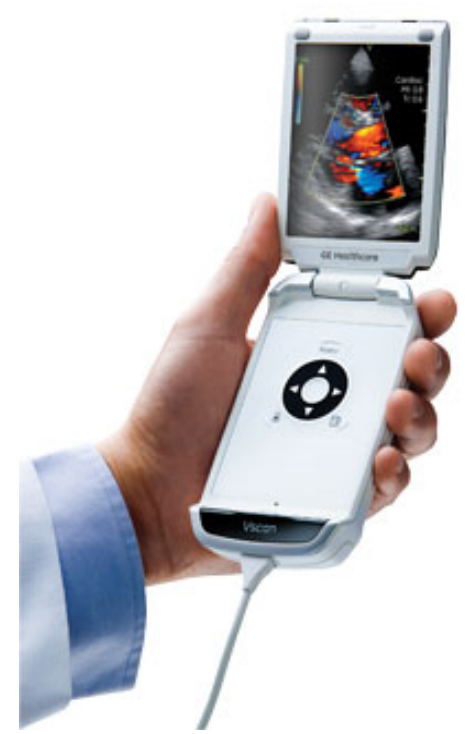

Figure 1. Hand-held Ultrasound Machine, contains an image of a portable hand-held ultrasound machine that is one of many types of ultrasound machines that can be used to perform POCUS examinations
Table 1. lists several advantages of utilizing the Point of Care Ultrasound in daily evaluations of patients. Some of the biggest advantages include the increased positive patient outcomes, increased efficiency of patient care (such as reduced time to diagnosis patient with POCUS) and can be performed next to a patient instead of having to transport the patient to a radiologic area

\section{Benefits of POCUS}

Reduced medical costs

Can be performed by medical professionals that has had POCUS training

Several internal organs can be assessed and scrutinized with POCUS

POCUS can be performed quickly

POCUS can be performed by any outpatient or inpatient bedside

POCUS helps physicians quickly diagnosis patients

POCUS allows more patients to be seen and treated

Increased patient care outcomes

\section{Discussion}

Point of Care Ultrasound is being used more and more throughout the world and America. This efficient procedure allows for several benefits for the medical facility as well as the patients. One of the largest advantages of POCUS is that it allows for the same area of treatment instead of having patients transported into different departments and having to wait on specialty examinations. POCUS therefore increases the speed of the overall treatment and diagnosis of patients. POCUS is breaking medical barriers by bringing specialty care to patients where they are located to help with preventative care, observing, and checkups (“The benefits of point," 2011).

\section{POCUS Defined}

Point of Care Ultrasound is a diagnostic tool that consists of medical personnel performing a specific ultrasound examination. POCUS is performed as a tool that a physician (or another medical

${ }^{\star}$ Correspondence to: Delores Jones, Registered Diagnostic Medical Sonographer from American Registry of Diagnostic Medical Sonography, University of Phoenix, USA, Tel: 301-7388401, E-mail: delolo1979@yahoo.com

Key words: focus care, POCUS, Point of Care Ultrasound, ultrasound trends

Received: May 31, 2019; Accepted: June 20, 2019; Published: June 27, 2019 
professional) may use to help determine where a person's symptoms are coming from. POCUS is especially helpful when a clinical examination is unclear to where problems are arising from. This examination usually consists of specific ultrasound protocols that are performed right next to the patient. POCUS is different from other ultrasounds in the sense that it can be used by any professional that is trained with POCUS (whereas other ultrasounds are predominately performed by trained Sonographers and interpreted by radiologists). It is important to note that the length of time of training for POCUS is shorter than traditional ultrasound training. In addition to that Point of Care Ultrasound can be utilized in a variety of settings for a variety of functions. This procedure can be used to monitor, investigate, or preventative care. As with all ultrasounds, it can be performed with a variety of ultrasound machines from small to great. The same is true with POCUS equipment. Handheld, portable, or traditional ultrasound machines can be utilized with this specific examination ("Point of care," n.d.). Figure 1 illustrates the appearance of a modern hand-held ultrasound machine that can be used to obtain images of the internal organs of a patient [1-5].

\section{POCUS in Emergency Medical Services}

POCUS is often utilized in emergency medical services. Point of Care Ultrasound allows for more efficient emergency medical services. Often many healthcare facilities utilize this ultrasound protocol in triage and emergency rooms. POCUS allows physicians to monitor a patient while trying to diagnosis the cause of their medical symptoms. POCUS quickly gives general ultrasound insight which minimizes wait time, costs, and hospital stay. Table 2 summarizes many of the emergency medical services that can utilize POCUS. Some of the areas listed include intensive care units, emergency room, acute pediatric settings, helicopter emergency medical services, and for natural disasters ("The benefits of point," 2011).

An example of POCUS that could be used in an emergency setting is cardiac ultrasound. Though a specific ultrasound cardiac scan, physicians or similar medical personnel could look at the ventricles and look at the functions of the vessels going to the heart (such as inferior vena cava or aorta). Additionally, through the use of POCUS, physicians can determine if there is any fluid around the heart which is called pericardial effusion. There are several POCUS examinations that can be performed in an emergency setting that can help doctors quickly save lives or better treat a patient.

\section{POCUS in Therapeutic and Monitoring}

There are several applications of Point of Care Ultrasound in areas of monitoring patients. Many medical facilities have observational areas to watch patients after surgery or to monitor a severe condition. POCUS is a great way to quickly assess how a patient is doing or healing. Besides monitoring, POCUS can be used in a variety of therapeutic procedures. Some therapeutic procedures include punctures, thoracentesis, paracentesis, aspirations, placement of various medical tools within the body (such as tubes), and foreign body removal. Figure 2 gives a quick summary of several therapeutic examinations that can be performed with the use of POCUS. Note that all of the procedures can be performed at bedside of the patient instead of with traditional

Table 2. POCUS in EMS. POCUS in EMS (emergency medical services) is utilized in a variety of areas. Some of the areas in this table includes the hospital, ICUs, Natural disasters, helicopter emergency services, emergency rooms, and acute pediatric services

\begin{tabular}{|c|c|c|}
\hline \multicolumn{3}{|c|}{ POCUS in EMS } \\
\hline Acute pediatric services & Helicopter emergency services & Natural disasters \\
\hline Emergency rooms & Intensive care units (ICUs) & Hospitals \\
\hline
\end{tabular}

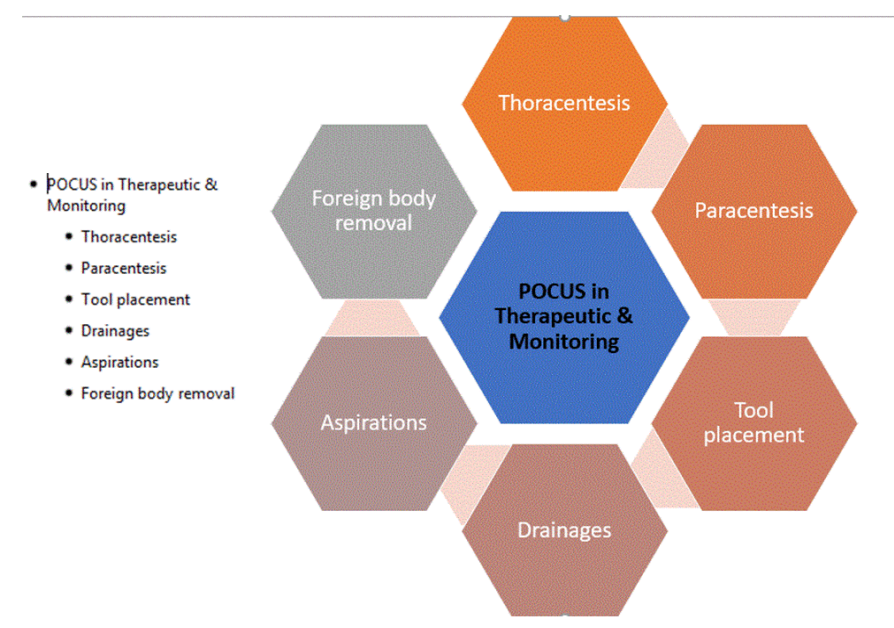

Figure 2. POCUS in Therapeutic \& Monitoring, lists some of the examinations that can be performed with POCUS in therapeutic applications. Additionally, POCUS is often performed in monitoring situations to confirm that a patient is healing or recovery as they should (and no complications is occurring)

ultrasounds (when patients have to be transported to a radiologic area to have the ultrasound performed by a sonographer). Additionally, POCUS can be used in any guided medical procedures to help surgeons stay in the area that they need to be in during the procedure ("Point of care," n.d.).

\section{POCUS in Outpatient Settings}

Patient settings or emergency care, POCUS can be used for increased efficiency in outpatient settings. Outpatient settings can utilize POCUS for preventative care as well as for diagnosing patients. POCUS reduces costs while quickly allowing patients to be seen by doctors in outpatient settings. The diagnosis accuracy of outpatients is increased with the utilization of POCUS. Examples of body parts that can quickly be assessed with Point of Care Ultrasound includes cardiac, abdominal, vascular, obstetrics, gynecological, musculoskeletal, and a variety of other areas. One specific example of a POCUS examination that can be performed in an outpatient setting is to see if there are any abnormalities on the thyroid. A quick scan of the thyroid performed by a physician can quickly determine if any abnormalities are going on within the organ. Another example of a POCUS exam in an outpatient center is DVT ultrasound exam. This exam looks at the vessels within the leg of a patient to quickly determine if there is a blood clot present causing the patient leg pain. There are many more parts of the body that can be scrutinized with the use of a POCUS exam [6-10].

\section{Conclusion}

Point of Care Ultrasound helps the health care industry provide better treatment for patients without the years of training usually required to perform and interpret ultrasounds. The accuracy and overall productivity of POCUS have caused this protocol to be used throughout America and the world. Health care is always changing, especially with the developments of new technologies. Speed of care is one area that many medical facilities are focusing on. Speed of care is important because it allows a decreased death rate and a better overall patient outcome if patients can be diagnosed and treated in a prompt. POCUS allows increased speed of care so that more patients can be seen by physicians in emergency and outpatient settings for a variety of exams. 


\section{References}

1. Bornemann P and Baretto T (2018) Point-of-care ultrasonography in family medicine. American Family Physician 98: 200-202.

2. Melgarejo S, Schaub A and Noble VE (2017) Point of care ultrasound: An overview. American College of Cardiology 27.
3. National Institute of Biomedical Engineering (2016) (NIH).

4. Point of care ultrasound. (PCOS) 123sonography.

5. The benefits of point-of-care ultrasound (2011) Healthcare-in-europe.com.

Copyright: (2019 Jones D. This is an open-access article distributed under the terms of the Creative Commons Attribution License, which permits unrestricted use, distribution, and reproduction in any medium, provided the original author and source are credited. 